\title{
Molecular Dynamics Simulation Studies on Effects of Erythritol on Alcohol Dehydrogenase Folding
}

\author{
Surya Prakash Dwivedi ${ }^{1}$, Neeraja Dwivedi ${ }^{1}$, Abhishek Tyagi ${ }^{1,3}$, R.B. Singh ${ }^{2}$ and Sanjay Mishra ${ }^{1,4, *}$ \\ ${ }^{I}$ Department of Biotechnology, College of Engineering \& Technology, IFTM Campus, Lodhipur Rajput, Delhi Road, \\ Moradabad 244 001, U.P., India \\ ${ }^{2}$ Halberg Hospital and Research Institute, Civil Lines Moradabad 244 001, U.P., India \\ ${ }^{3}$ Indian Institute of Technology, Kharagpur 721304, West Bengal, India \\ ${ }^{4}$ Department of Biotechnology \& Microbiology, Institute of Foreign Trade \& Management, Lodhipur Rajput, Delhi \\ Road, Moradabad 244001, U.P., India
}

\begin{abstract}
Liver Alcohol dehydrogenase (LADH) catalyzes reversible oxidation of primary and secondary alcohols to aldehyde using $\mathrm{NADH}^{+}$as a coenzyme. The aim of the present study was to validate preexisting biophysical characterization of erythritol using simulation studies of protein-erythritol-water interaction in solution at the molecular level. Two molecular dynamics simulations of the protein $\mathrm{ADH}$ in solution at room temperature were carried out, one in the presence (about $0.77 \mathrm{M}$ ) and another in the absence of erythritol. The erythritol molecules were observed to cluster and move toward the protein, and expel water from the protein surface and ultimately form hydrogen bonds with protein. Besides, the coating by erythritol was noticed to reduce the conformational fluctuations of the protein compared to the erythritolfree system. Conclusively, at a moderate concentration of erythritol solution, erythritol molecules cluster in the protein region and interact with $\mathrm{ADH}$ via many $\mathrm{H}$-bonds that prevent the protein folding, although the data shown in the present study are contrary probably due to providing a minimum solution from water with an osmolyte like erythritol with $10 \mathrm{~ns}$ simulation and subsequently studying the desired interaction with protein using above solution using $15 \mathrm{~ns}$ of simulation. Over findings provide new insights into further exploration of the studies pertaining to ADH activity in alcoholic liver diseases leading to lever Cirrhosis.
\end{abstract}

Keywords: ADH, erythritol, molecular dynamics simulation, H-bonds, protein folding.

\section{INTRODUCTION}

Erythritol (2R, 3S)-butane-1, 2, 3, 4-tetraol) is a natural sugar alcohol, a sugar substitute [1]. It occurs naturally in fruits and fermented foods. Industrially, it is produced from glucose by fermentation with yeast, Moniliella pollinis [2]. Horse liver alcohol dehydrogenase (HLADH, EC 1.1.1.1) catalyzes reversible oxidation of primary and secondary alcohols to aldehyde using $\mathrm{NADH}^{+}$as a coenzyme. The oxidation involves alcohol hydroxyl proton ionization and passage of the $\mathrm{H}^{+}$through a hydrogen bond network to water via His51 imidazole and hydride transfer from alkoxide to $\mathrm{NAD}^{+}$. This enzyme has a molecular weight of $80 \mathrm{kDa}$ and is a dimer of two identical subunits as reported in the X-ray structure. The enzyme has a twelve-stranded, $\beta$ - sheet, which makes up the central core of the dimer. Each subunit of this dimeric enzyme binds one molecule of $\mathrm{NAD}^{+}$and two $\mathrm{Zn}$ (II) ions. One of the zinc ions is in the active site, while another is structural. Upon enzyme substrate complex formation a rigid body rotation closes the cleft between the coenzyme and catalytic domains. This conformational change is

*Address correspondence to this author at the Department of Biotechnology \& Dean (Research) College of Engineering \& Technology, IFTM Campus, Lodhipur Rajpoot, Delhi Road, Moradabad (U.P.) India; Tel: +91 0591 2360817; Fax: +91 0591 2360818; E-mail: sanjaymishra66@gmail.com described by a rotation of $9^{\circ}$ to $10^{\circ}$ in the monoclinic form. The hydroxyl oxygen of the alcohol substrate is ligated to the active site zinc.

One of the common methods in industry for stabilizing and keeping of enzymes is use of osmolytes. Osmolytes are compounds that support proteins and cells in unfavorable condition. These materials are usually small and non-charge organic compounds that don't change enzymatic activity. Besides, osmolytes can act as molecular chaperon and can enumerate suitable compound for trapping molten globule of some proteins. Nevertheless, pattern of melting of helixes can be changed accompanied with amplification of hydrophobic interaction, and hydrogen bonds. Osmolytes are small organic compound that are ubiquitous in living systems. Osmolyte molecules in aqueous solution can have profound effects on protein stability, structure, and function [3], and it has long been known that osmolytes as solvent additives have significant effects on protein folding or unfolding [4]. Various osmolytes such as trimethylamine $\mathrm{N}$-oxide [5], glycerol [6], betaine [7], and erythritol [8] were used to study protein folding experimentally. An osmolyte can enhance and inhibit protein folding, and the favoring and inhibiting effects of an osmolyte on protein folding depend on its concentration. Despite its widespread use, the molecular basis for osmolyte's ability to assist and inhibit protein folding 
remains unknown. It is reported that an osmolyte stabilizes a folded state of protein, while it binds to the protein by hydrogen bonds. The stabilizing effect shifts the equilibrium toward the native state, thereby favors the protein folding [9]. It has been mentioned that an osmolyte directly influences protein folding by being preferentially excluded from the backbone of the protein, which raises the free energy of the unfolded state and favors the folded population [10]. In fact, protein stability is the result of a balance between the intramolecular interactions of protein functional groups and their interactions with the solvent environment. Thus, as mentioned above, presence of small organic molecules like osmolyte in aqueous solution can also have basic effects on protein stability, structure, and function.

The influence of osmolytes on biomolecules has been extensively investigated. Currently available experimental techniques have often led to contradictory conclusions. Three main hypotheses have been put forward involving (1) the direct interaction between osmolyte molecules and the protected biostructure through hydrogen bonds (water-replacing hypotheses) [11-14]; (2) the trapping of water molecules close to the biomolecular surface (water-layer hypotheses)[15]; and (3) the entrapment of a particular biomolecu;ar conformation in a high-viscosity osmolyte glass (mechanical-entrapment hypotheses) [16, 17].

Theoretically, it revealed that trehalose could enhance and inhibit protein folding and the favoring and inhibiting effect of trehalose on protein folding depends on its concentration [18]. At higher trehalose concentrations, trehalose prevents the peptide from folding to its native structure. In another theoretical study, it is reported that at moderately concentrated trehalose solution, trehalose does not reduce the conformational fluctuation of the protein [19]. It is apparent from mentioned study [20] that in lower concentration of an osmolyte such as trehalose, the peptide and protein folds faster than that in water. Molecular-level insights into how osmolyte molecules effect protein folding would be invaluable for the rational design of small molecular additives for enhancing or hindering the folding of protein. However, it seems unlikely that experimental approaches can provide the molecular details. Molecular simulation is expected to contribute greatly to this end. The aim of the present study was to: (a) validate preexisting biophysical characterization of erythritol using simulation studies of protein-erythritol-water interaction in solution at the molecular level; and (b) provide new insights into further exploration of the studies pertaining to ADH activity in alcoholic liver diseases leading to lever Cirrhosis.

\section{MATERIALS AND METHODS}

All molecular dynamics (MD) simulations were performed using the gromacs 3.3 program together with the G43a1 force field [20-23] for each simulation. The MD simulations were carried out by particle mesh Ewald method [24] for the electrostatic interactions. The Vander Waals cutoff was 14 $\AA$. The integration time step was 1 femtosecond (fs), with the neighbor list being updated every fifth step by using the grid option and a cutoff distance of $12 \AA$. Periodic boundary condition was used with constant number of particles in the systems, constant pressure, and constant temperature simulation criteria (NPT). In this simulation the systems were coupled to external constant temperature $(100,200,300 \mathrm{~K}, \tau=0.1$ $\mathrm{ps})$ in three steps and external constant pressure bath (1 atm, $\tau=0.5 \mathrm{ps})$. For all simulations, the water molecules were added as a simple point charge (SPC) model.

The force field parameters for the erythritol were extracted from the website pertaining to Dundee PRODRG Server [25]. Besides, force field parameters of glucose were positively considered for the simulation studies. Protonated state of various residues were determined from the $\mathrm{pK}_{\mathrm{a}}$ calculation by PROPKA at $\mathrm{PH}=7$ [26]; and the point charges for erythritol was obtained from a HF/6-31G* single point calculation in Gaussian 98 using the CHELPG fitting procedure [27-28]. Note that the limitation of this approach is that the polarization effect associated with the condensed phase environment is not explicitly included, although the tendency for the HF/6-31G* QM level of theory to overestimate dipole moments has been suggested to account for this deficiency [29].

\section{RESULTS AND DISCUSSION}

In the present study the attempts have been made to determine effect of erythritol on folding of $\mathrm{ADH}$, and therefore ADH had to be studied in a box including water molecule and ions in absence and presence of erythritol with a moderate concentration. Initially, a mixture of water and erythritol molecules was prepared considering it in a minimum location, followed by filling latter solution in a box involving ADH. Ultimately, equilibrium and minima geometries of ADH could be obtained in a box with water molecule and ions both in absence and presence of erythritol. The data extracted from the present studies are summarized as follows:

For the Preparation of a minimum solution of water molecules with erythritol molecules, an erythritol molecule was first placed in a cubic box with periodic boundary conditions. The size of the cubic box was $50 \AA$. Then, 65 molecules of erythritol were added to the box. Ultimately, the box was filled with 3491 SPC water molecules. Since this system is neutral, addition of ions is not required. Referring to erythritol density, its molecular weight and ratio between the number of erythritol molecules and that of water molecules which is equal to $\sim 1 / 54$, erythritol concentration would be about $0.95 \mathrm{M}$ in the box. Thus, erythritol has a moderate concentration. Note that erythritol molecules were randomly added to the aforementioned box [18]. Initially, the water molecules alone were subjected to energy minimization with the erythritol molecules kept fixed in their initial configuration. The water molecules were then allowed to evolve using a molecular dynamics simulation for 20 ps with a step time of $1 \mathrm{fs}$, again keeping the structure of the erythritol molecules fixed. Next, the entire system was minimized using the steepest descent of 1000 steps followed by conjugate gradients of 9000 steps. In order to obtain equilibrium geometry at $300 \mathrm{~K}$ and $1 \mathrm{~atm}$, the system was heated at a weak temperature $(\tau=0.1 \mathrm{ps})$ and pressure $(\tau=0.5 \mathrm{ps})$ coupling by taking advantage of the Berendesen algorithms [23]. Heating time for molecular dynamics simulations at $100 \mathrm{~K}, 200 \mathrm{~K}$ and $300 \mathrm{~K}$ was 100 ps. All above simulations were performed at constant temperature and pressure with a nonbonded cutoff of $14 \AA$. Molecular dynamics simulation was further carried out for $3 \mathrm{~ns}$ at $300 \mathrm{~K}$, followed by structural minimization calculation. The latter minimization was per- 
formed at the steepest descent of 1000 steps followed by conjugate gradients of 9000 steps. This way, a minimum geometry was obtained for solution of erythritol with water molecules. Variation in kinetics and potential energy components versus time in MD simulation has been well illustrated (Figs. 1a \& b). By having a look at this figure, it reveals that the kinetics and potential energy components would be expected to fluctuate in equal and opposite direction. This point is explicitly specified by referring to Fig. (1b). Data of afore mentioned Figure belongs to last 300 ps of simulation. Latter facts show that energy conservation is satisfied in MD simulation performed [30]. Thus, the simulation has been done accurately and the extracted equilibrium structure was obtained by employing accurate algorithms and parameters in solving motion equations and under energy stability conditions.

As mentioned elsewhere, the ability to climb over energy barrier is limited in MD simulation. Molecular dynamics is used to explore the conformational space in order to find a conformation (or conformations) that only have a low intrinsic energy. Therefore, it often attempts to search conformational space. In fact, conformational analysis gives us a strong chance to pass probable barrier energies in phase space and reach to more deep minima of a solution studied. Simulated annealing is often a convenient way to ensure the conformational space is explored effectively [31], and that in which the conformational space is sampled at sufficiently high temperatures. A simulated annealing algorithm starts from a given geometry and has an ability to cross barrier to other conformers.

In simulated annealing, starting point has been the final system obtained after $3 \mathrm{~ns}$ MD simulation. First, mentioned system has been heated to $600 \mathrm{~K}$ by employing of an MD simulation of 200ps. Strategy being decrease in temperature from $600 \mathrm{~K}$ to $300 \mathrm{~K}$ in time duration of $200 \mathrm{ps}$, followed by keeping temperature in $300 \mathrm{~K}$ for $200 \mathrm{ps}$. Then, temperature was decreased from $300 \mathrm{~K}$ to $50 \mathrm{~K}$ followed by increase from $50 \mathrm{~K}$ back to $300 \mathrm{~K}$. Time duration for each increase or decrease of temperatures was $50 \mathrm{ps}$. This way carrying out a simulated annealing of $7 \mathrm{~ns}, 10$ near local minima structures (10 geometries in $50 \mathrm{~K})$ is resulted form starting system. Inspection of the annealed systems showed no indication of any bond breakage or tension in structures as expected. U1timately, the annealed near local geometries were optimized
- - - - Kinetic Energy
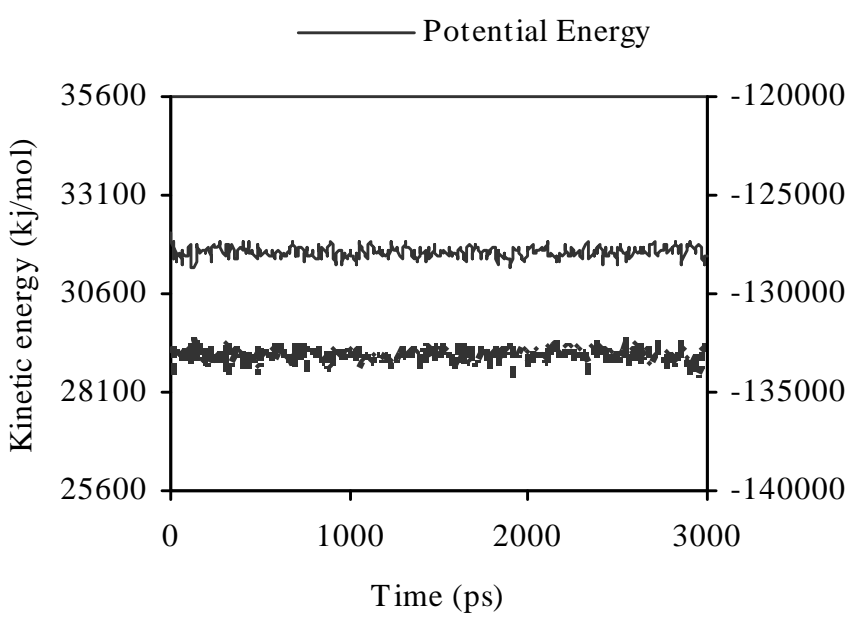

- - - - Kinetic energy $(\mathrm{kj} / \mathrm{mol})$

Potential energy $(\mathrm{kj} / \mathrm{mol})$

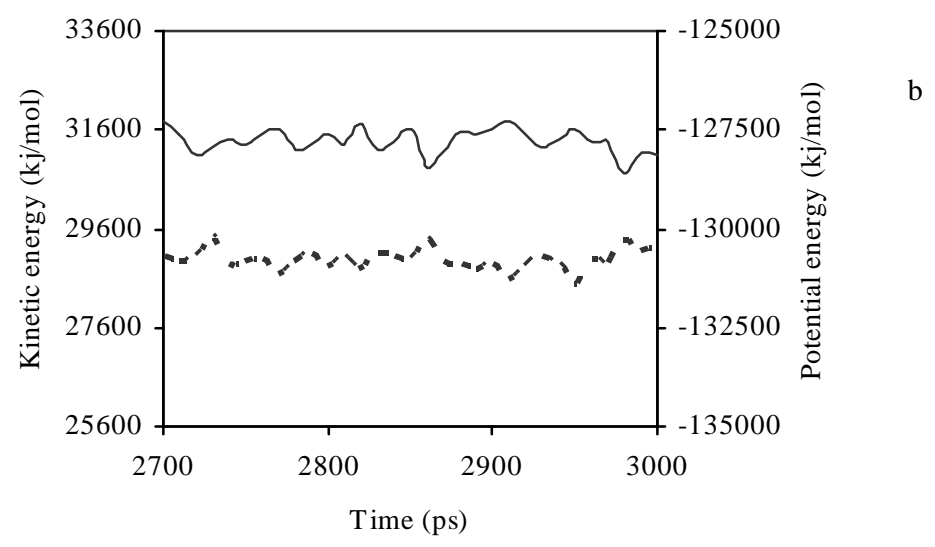

Fig. (1). Variation in kinetic and potential energies components observed in MD simulation of a box including erythritol and water molecules at $300 \mathrm{~K}$. 
by taking advantage of steepest descent of 1000 steps followed by conjugate gradients of 9000 steps methods. Among these minima geometries, a geometry was chosen which had the least MM energy value. Energy of the latter geometry was even less than that of the one which had been obtained from optimization of system after MD simulation of $3 \mathrm{~ns}$. Thus, in order to obtain a minimum structure from solution of erythritol with water molecules, performing conformational analysis is necessary.

As mentioned above, a minimum geometry of solution involving erythritol and water molecules was obtained using MD simulation as well as simulated annealing. In order to investigate erythritol's effect on ADH stability, performing two simulations are required, namely simulation of $\mathrm{ADH}$ in a box including water molecules and ions in the absence as well as in the presence of erythritol molecules. The X-ray structure of ADH (HLADH, EC 1.1.1.1) was extracted from protein data bank. The latter structure was placed in a solvent box with $60683 \mathrm{spc} 216$ water molecules being equal to a $1.8 \AA$ distance for ADH to the box edges. Then, same Xray structure was placed in a cubic box. The size of the cubic box was $110 \AA, 110 \AA, 160 \AA$. The latter box is filled with erythritol and water molecules belonging to minimum solution which had been obtained from previous step. In this way, two systems were thus prepared: one including $\mathrm{ADH}$ and 60683 SPC water molecules, while another involving ADH and 45272 SPC water molecules as well as 689 erythritol molecules. Neutralization of each system required addition of eight $\mathrm{Cl}^{-}$ions. It is worth mentioning that use of cubic box in the study of effect of osmolytes on protein is benefited in previous works $[18,19]$.

Referring to erythritol density, its molecular weight and ratio between the number of erythritol molecules and that of water molecules which is equal $\sim 1 / 66$, erythritol concentration would be about $0.77 \mathrm{M}$ in the box. Thus, erythritol has a moderate concentration. Initially, former system namely the water molecules and $\mathrm{Cl}^{-}$ions alone were subjected to energy minimization with the ADH kept fixed. Next, latter system, the water molecules, $\mathrm{Cl}^{-}$ions as well as erythritol molecules were exposed to energy minimization with the ADH remained fixed. Both systems along with their corresponding constituents were then allowed to evolve using a molecular dynamics simulation for 20 ps with a step time of $1 \mathrm{fs}$, again keeping the structure of the ADH molecules fixed. Subsequently, following operations were performed for two systems studied.

Each of the entire above two systems was individually minimized using the steepest descent of 1000 steps followed by conjugate gradients of 9000 steps without imposing any constraint. In order to obtain equilibrium geometry at $300 \mathrm{~K}$ and $1 \mathrm{~atm}$, the system was heated at a weak temperature ( $\tau=$ $0.1 \mathrm{ps})$ and pressure ( $\tau=0.5 \mathrm{ps})$ coupling by taking advantage of the Berendesen algorithms [16]. Heating time for molecular dynamics simulations at $100 \mathrm{~K}, 200 \mathrm{~K}$ and $300 \mathrm{~K}$ was 100 ps. All above simulations were performed at constant temperature and pressure with a non-bonded cutoff of 14 A. A molecular dynamics simulation was performed for 5 $\mathrm{ns}$ at $300 \mathrm{~K}$. Ultimately, another molecular dynamics simulation was further carried out for $10 \mathrm{~ns}$ at $300 \mathrm{~K}$, followed by structural minimization calculation. The latter minimization was performed at the steepest descent of 1000 steps followed by conjugate gradients of 9000 steps. This way, a minimum geometry was obtained for $\mathrm{ADH}$ at moderately concentrated erythritol solution. As far as variations in total energy versus time is concerned, for $10 \mathrm{~ns}$ of the MD simulations is about less than 1 part in 1000 and it is quite apparent that the kinetics and potential energy components would be expected to fluctuate in equal and opposite direction for MD simulation of $\mathrm{ADH}$ in absence and presence of erythritol molecules. Latter facts show that law of energy conservation is fulfilled in MD simulations [29]. Thus, the simulation has been done accurately and the extracted equilibrium structures were obtained by benefitting accurate algorithms and parameters in solving motion equations and under energy stability conditions. In addition, temperature fluctuation for the simulation at $300 \mathrm{~K}$ for each of the systems was less than $0.5 \mathrm{~K}$. Therefore, the final equilibrium structure at $300 \mathrm{~K}$ belonging to every system was obtained under temperature stability conditions.

Data obtained from the experiments carried out on conformational changes of the ADH in absence and presence of erythritol molecules entails that the RMSD from the initial structure for backbone atoms is used to represent the conformational changes of the $\mathrm{ADH}$ during the folding. The Backbone RMSD is displayed as a function of time in Fig. (2), during simulation of $\mathrm{ADH}$ in absence and presence of erythritol molecules. Having a look at Fig. (2), it reveals the final values of the Backbone RMSD as well as these values on the average are decreased in presence of erythritol molecules, and they are smaller than that in pure water. This point is obvious from values of Backbone RMSD during simulation and belonging to last 300ps of simulations which has been decreased about $5 \%$ and $6 \%$ in presence of erythritol molecules, respectively. Besides, fluctuations in aforementioned values are considerably weakened by $\sim 16 \%$ and $37 \%$ in presence of erythritol molecules than that in pure water, respectively. Latter fact has been clarified by referring to standard deviation for RMSD values which are equal to 0.02 and 0.017 and RMSD values belonging to last 300ps of that are equal to 0.0095 and 0.006 in absence and presence of erythritol molecules in last 300ps of simulation, respectively.

Besides, the RMSF per residues show that the mobility of residues in the presence of the erythritol molecules is decreased by $\sim \%$, when compared with the mobility in the absence of erythritol molecules. Standard deviation for theses RMSF values are equal to about 0.041 and 0.031 in absence and presence of erythritol molecules, respectively. Thus, fluctuations in these RMSF are considerably decreased by $\sim 22 \%$ in presence of erythritol molecules than that in pure water. Also, mobility of some residues is considerably in the presence of erythritol molecules. As examples, the RMSF values belonging to residues 1-45 of ADH have been decreased $\sim 20$ on the average due to presence of erythritol molecules. Also, fluctuations in these RMSF are considerably weakened by $\sim 42 \%$ in presence of erythritol molecules than that in pure water. Latter point is completely clear from standard deviation for theses RMSD values which are equal to 0.075 and 0.043 in absence and presence of erythritol molecules, respectively.

Considering the RMSF per atoms, it is obvious that the mobility of atoms belonging to ADH have been reduced in the presence of erythritol molecules $\sim 7 \%$ as observed for RMSF 
— In the absence of erythritol molecules (a)
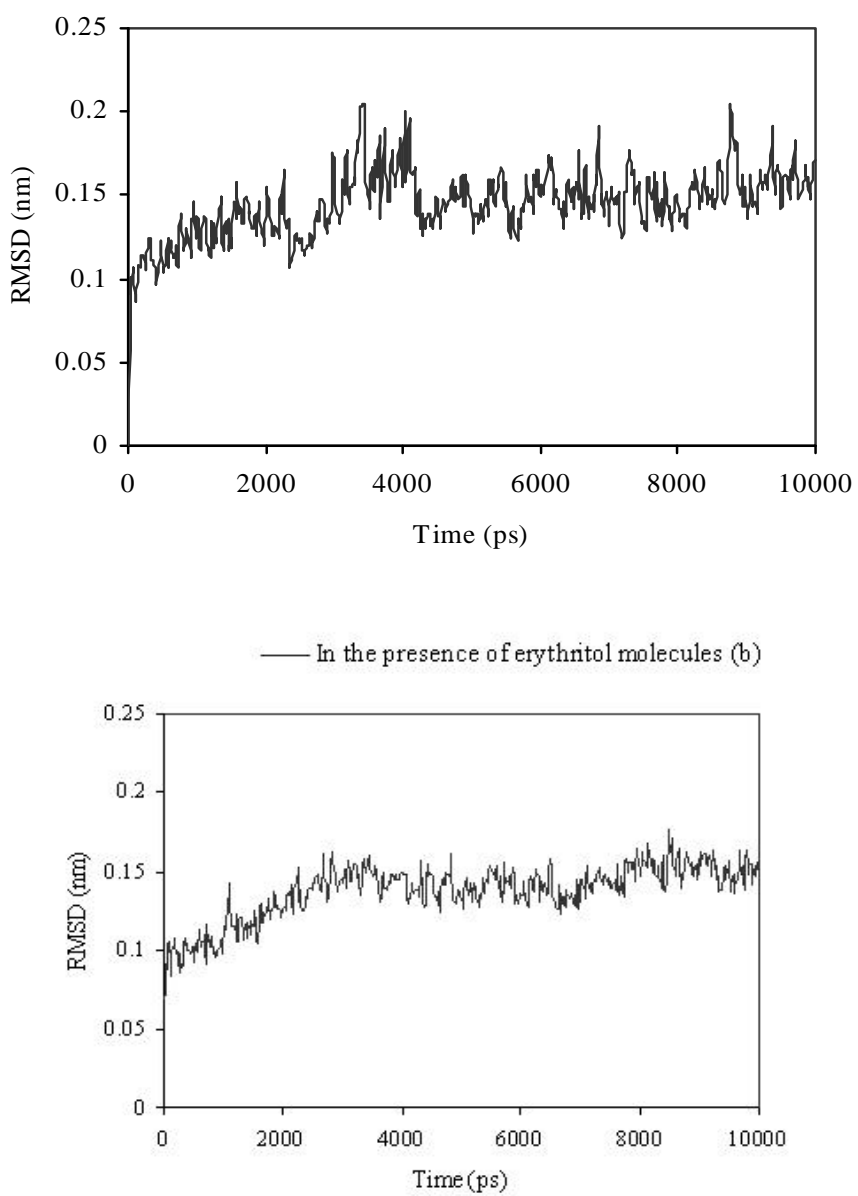

Fig. (2). Root-mean-square atomic positional deviation (RMSD) obtained from the initial structure for Backbone atoms (a) in the absence of erythritol molecules (b) in the presence of erythritol molecules.

per residues. This reduction is completely figured out by referring to atoms 1-431 which belong to residues 1-45. These atoms have a mobility decrease of $\sim 16 \%$ in moderately concentrated erythritol solution relative to the atoms of $\mathrm{ADH}$ in pure water solvent. Further, RMS deviation of residues and atoms belonging to ADH is decreased in the presence of erythritol molecules. These findings completely corroborate to RMSD Backbone values obtained and summarized in Fig. (3). Thus, the results depicted above implicate that erythritol inhibits the folding of $\mathrm{ADH}$ at moderate concentration of erythritol solution. Such a behavior is qualitatively consistent with the experimental observation of firefly luciferase refolding in presence of erythritol [32], and a speculative theory about $\beta$-hairpin folding [11].

The ADH folding via $\mathrm{H}$-bond interactions involved in the absence and presence of erythritol molecules has been analyzed. Table 1 lists the total number of inter-molecular and intra-molecular H-bonds for final geometries of ADH which were obtained from MD simulations of $15 \mathrm{~ns}$ in the absence and presence of erythritol molecules. It has been known that backbone H-bonds increase during the folding [33]. The backbone $\mathrm{H}$-bonds are closely connected during the peptide desolvation, because $\mathrm{H}$-bond between water and the ami- dogen and carboxyl groups must be broken before the backbone $\mathrm{H}$-bond can be formed. In fact peptide desolvation is a deriving force in peptide folding [34]. Data of Table 1 show that intra-molecular $\mathrm{H}$-bonds belonging to $\mathrm{ADH}$ as well as inter-molecular $\mathrm{H}$-bonds between $\mathrm{ADH}$ and water molecules are decreased in presence of erythritol molecules about $4 \%$ and $19 \%$, respectively. Also, some $\mathrm{H}$-bonds are formed between $\mathrm{ADH}$ and erythritol molecules. Thus, it can be concluded that erythritol molecules accumulate around the protein and exclude water. Besides, forming $\mathrm{H}$-bonds between protein and erythritol has an effect on the formation of the protein backbone H-bonds. Thus, although erythritol molecules can dissolve protein, but formation of more $\mathrm{H}$-bond between erythritol and ADH can act a factor for inhibition of folding protein, while backbone $\mathrm{H}$-bonds is also changed. Ultimately, the protein cannot fold into its native state in moderately concentrated erythritol solution due to the direct interaction between osmolyte molecules and the protected biostructure through hydrogen bonds (water-replacing hypotheses). Nevertheless, at a moderate concentration of erythritol, more erythritol-erythritol interaction occurs (Table 1). This is because of clustering of most of the erythritol molecules in the simulation system. Nevertheless, the num- 

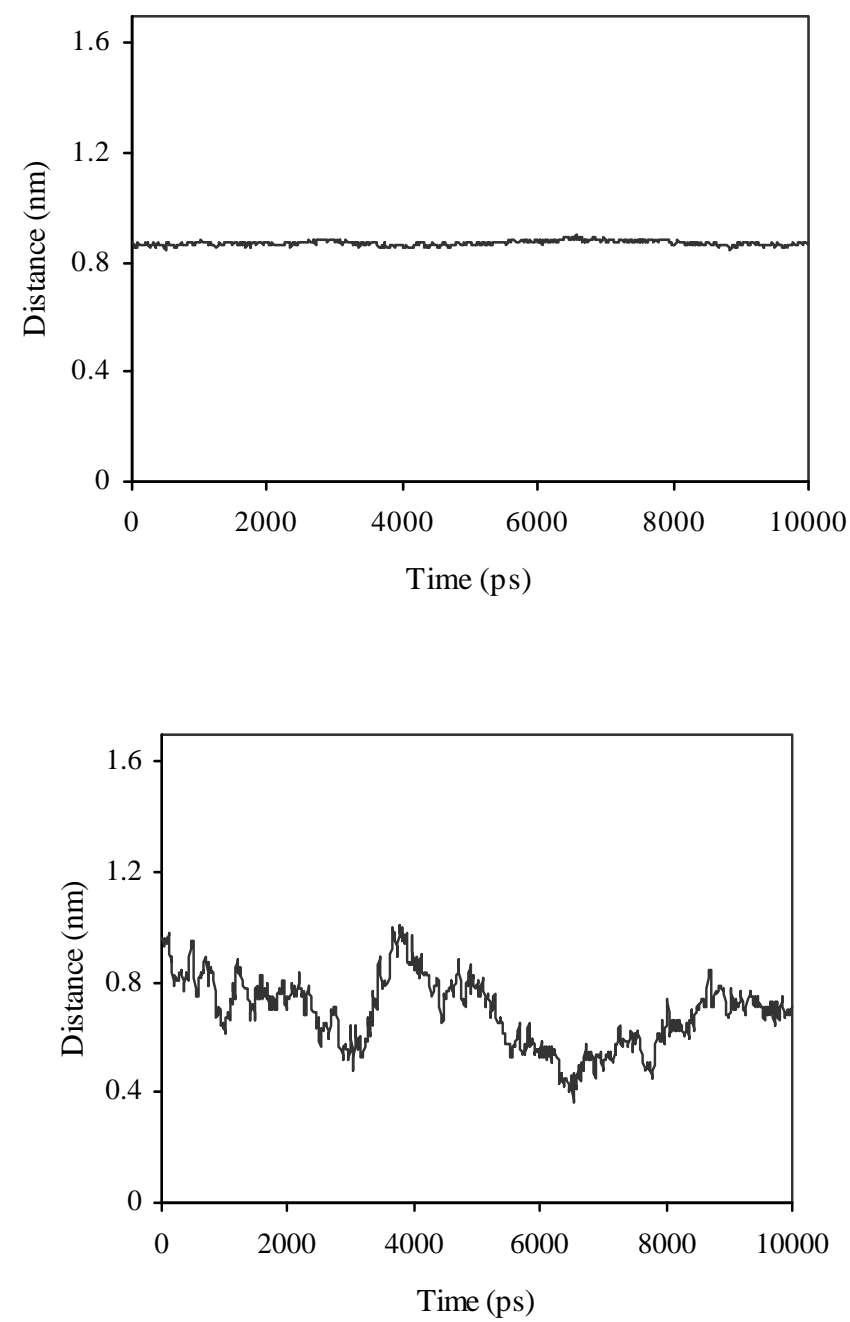

Fig. (3). The average distances of the water (a) and erythritol (b) molecules with the center of geometry of the protein as a function of time for the simulation including erythritol molecules.

Table 1. The Number of Inter-Molecular and Intra-Molecular Hydrogen Bonds for Conclusive Geometries of ADH Obtained from MD Simulations of Femtosecond in the Absence and Presence of Erythritol Molecules

\begin{tabular}{|l|c|c|}
\hline Number of Hydrogen Bonds & Absence of Erythritol Molecules & Presence of Erythritol Molecules \\
\hline \hline Water-ADH & 1056 & 852 \\
\hline ADH-ADH & 563 & 541 \\
\hline Water-erythritol & ND & 4658 \\
\hline Erythritol-ADH & ND & 214 \\
\hline Erythritol-Erythritol & ND & 754 \\
\hline
\end{tabular}

$\mathrm{ND}=$ not detected.

ber of hydrogen bonds between $\mathrm{ADH}$ and water molecules as well as the number of hydrogen bonds between erythritol and water molecules is decreased during simulation, respectively and number of hydrogen bonds between ADH and erythritol molecules is increased during simulation (Table 1). Consequently, more $\mathrm{H}$-bonds can form between the protein and erythritol molecules when protein folds in the presence of moderately concentrated solution of erythritol. Also, the average distances of the water and erythritol molecules with the center of geometry of the protein are displayed in Fig. (3) for simulation including erythritol molecules.

\section{CONCLUSION}

Conclusively, the major findings from the present study provide new insights into the development and projection of new design of putative molecular additives to exaplain com- 
prehensively the probable involvement of a novel mechanism of osmolysis of erythritol molecules, ultimately, leading to inhibiting the folding of alcohol dehydrogenase, an enzyme catalyzing a reversible oxidation of primary and secondary alcohols to aldehyde using $\mathrm{NADH}^{+}$as a coenzyme. Further work along these notions and obejectives is in progress in our laboratory.

\section{ACKNOWLEDGEMENTS}

The authors are grateful to Prof. R. M. Dubey (Managing Director) and Prof. A. Srivastav (Director), CET, IFTM, Moradabad, U.P, India for providing the necessary facilities and encouragement. Besides, Dr. R.S. Sangwan and Dr. (Mrs.) N.S. Sangwan (Senior Scientists, CIMAP, Lucknow India) are duly acknowledged for critical reading of the manuscript and valuable suggestions.

\section{REFERENCES}

[1] FDA/CFSAN: Agency Response Letter: GRAS Notice No. GRN 00076

[2] Shindou T, Sasaki Y, Miki H, Eguchi T, Hagiwara K, Ichikawa T. Determination of erythritol in fermented foods by high performance liquid chromatography. Shokuhin Eiseigaku Zasshi 1988; 29(6): 419-22.

[3] Rösgen J. Molecular basis of osmolyte effects on protein and metabolites. Methods Enzymol 2007; 428: 459-86.

[4] Bolen DW, Baskakov IV. The osmophobic effect: natural selection of a thermodynamic force in protein folding. J Mol Biol 2001; 310: 955-63.

[5] Bennion BJ, Daggett V. Concentration of Urea-induced protein denaturation by trimethylamine N-oxide: a chemical chaperone at atomic resolution. Proc Natl Acad Sci USA 2004; 101: 6433-38.

[6] Dong XY, Huang Y, Sun Y. Refolding kinetics of denaturedreduced lysozyme in the presence of folding acids. J Biotechnol 2004; 114: -42 .

[7] Bourot S, Sire O, Trautwetter A, et al. Glycine betaine-assisted protein folding in a lys A mutant of Escherichia coli. J Biol Chem 2000; 275: 1050-56.

[8] Chen LY, Ferreira JAB, Costa SMB, Cabrita GJ, Otzen DE, Melo EP. Compaction of ribosomal protein S6 by sucrose occurs only under native conditions. Biochemistry 2006; 45: 2189-99.

[9] Allison SD, Chang B, Randolph TW, Carpenter JF. Hydrogen bonding between sugar and protein is responsible for inhibition of dehydration-induced protein unfolding. Arch Biochem Biophys 1999; 365: 289-98.

[10] Auton M, Bolen DW. Additive transfer free energies of the peptide backbone unit that are independent of the model compound and the choice of concentration scale. Biochemistry 2004; 43: 1329-42.

[11] Prestrelaki S, Tedescji N, Arakawa T, Carpenter JF. Dehydration induced conformational transitions in proteins and their inhibition by stabilizers. Biophys J 1993; 65: 661-71.

[12] Carpenter JF, Crowe JH. An infrared spectroscopic study of the interaction of carbohydrates with dried protein. Biochemistry 1989; 28: 3916-22.

[13] Crowe JH, Carpenter JF, Crowe LM. The role of vitrification in adhydrobiosis. Annu Rev Physiol 1998; 6: 73-103.
[14] Allison SD, Chang B, Randolph TW, Carpenter JF. Hydrogen bonding between sugar and protein is responsible for inhibition of dehydration-induced protein unfolding. Arch Biochem Biophys 1999; 365: 289-98.

[15] Belton PS, Gil AM. IR and raman-spectroscopic studies of the interaction of erythritol with hen egg-white lysozyme. Biopolymers 1994; 34: 957-61.

[16] Ansari A, Jones CM, Henry ER, Hofrichter J, Eaton WA. The role of solvent viscosity in the dynamics of protein conformational changes. Science 1992; 256: 1796-98.

[17] Hagen SJ, Hofrichter J, Eaton WA. Protein reaction-kinetics in a room-temperature glasss. Science 1995; 269: 959-62.

[18] Fu-Feng Liu, Xiao-Yan Dong. Molecular mechanism for the effects of erythritol on $\beta$-hairpin folding revealed by molecular dynamics simulation. J Mol Graph Modell 2008; 27: 421-29.

[19] Lins D, Pereira S, Cristina H, Hünenberger P. Erythritol-protein interaction in aqueous solution, Protein: funct Bioinformatics. 2004; 55: 1777-86.

[20] Van der Spoel D, Lindahl E, Hess B, Groenhof G, Mark AE, Berendsen HJ. GROMACS: fast, flexible, and free. J Comp Chem 2005; 26: 1701-18.

[21] Bekker H, Berendsen HJC, Dijkstra EJ, et al. Gromacs method of virial calculation using a single sum. In Physics Computing 92 Singapore World Scientific. 1993.

[22] Lindahl E, Hess B, van der Spoel D. Gromacs 3.0: A package for molecular simulation and trajectory analysis. J Mol Mod 2001; 7: 306-17.

[23] Berendsen HJC, van der Spoel D, van Drunen R. GROMACS: A message-passing parallel molecular dynamics implementation. Comp Phys Commun 1995; 91: 43-56.

[24] Daren T, York D, Pedersen L. Partucle mesh ewald: an N log (N) method for ewald sums in large systems. J Chem Phys 1993; 98 : 1463- 72 .

[25] Schuettelkopf AW, van Aalten DMF. PRDRG: A tool for high throughout crystallography of protein-ligand complexes. Acta Crystallogr Sect D-Biol Crystallogr 2004; 60: 1355-63.

[26] Li H, Roberton AD, Jensen, JH. Very fast empirical prediction and rationalization of protein pKa values. Proteins 2005; 61: 704-21.

[27] Gleeson MP, Burton NA, Hillier IH. Prediction of the potency of inhibitors of adenosine deaminase by QM/MM calculations. Chem Commun 2003; 17: 2180-81.

[28] Breneman CM, Wiberg KB. Determining atom-centered monopoles from molecular electrostatic potentials: the need for high sampling density in formamide conformational analysis. J Comput Chem 1990; 11: 361-73.

[29] Hollup SM, Salensminde G, Reuter N. WEBnm @: a web application for normal mode analyses of proteins. BMC Bioinformatics 2005; 6: 52-9.

[30] Leach AR. Molecular Modelling, principles and applications. 2nd ed. Pearson Education EMA, Longman. 2001; pp 390.

[31] Leach AR. Molecular Modelling, principles and applications. 2nd ed. Pearson Education EMA, Longman 2001; p. 436.

[32] Singer MA, Lindquist S. Multiple effects of erythritol on protein folding in vitro and vivo. Mol Cell 1998; 1: 639-48.

[33] Scheiner S. Contributions of $\mathrm{NH}$...O and $\mathrm{CH}$... O hydrogen bonds to the stability of $\beta$-sheets in proteins. J Phys Chem B 2006; 110: 18670-9.

[34] Daidone I, Ulmschneider MB, Nola AD, Amadei A, Smith JC Dehydration-driven solvent exposure of hydrophobic surfaces as a driven force in peptide folding. Proc Natl Acad Sci USA 2007; 104: $15230-5$. 